ISSN: 1130-2887 - eISSN: 2340-4396

DOI: https://doi.org/10.14201/alh.21303

\title{
IMPACTO DE LA ELIMINACIÓN DE IMPUESTOS \\ SOBRE EL CONSUMO DE ALIMENTOS Y PRODUCTOS AGRÍCOLAS EN BRASIL
}

Impacts of a tax elimination on consumption of food and agricultural products in Brazil

\author{
Rayan WOLF (1) \\ Universidade Federal de Viçosa \\ $\triangle$ rayan.wol@@ufv.br
}

Abdoulaye Aboubacari MOHAMED
Universidade Federal de Viçosa
$\square$ aboubacari.abdoulaye@ufu.br

Fabrício Sepúlveda GOMES (1)

Universidade Federal de Viçosa

$\triangle$ fabricio.sepulveda@ufu.br

\author{
Angelo Costa GURGEL \\ Fundação Getulio Vargas \\ $\triangle$ angelo.gurgel@fgu.br \\ Erly Cardoso TEIXEIRA (1) \\ Universidade Federal de Viçosa \\ $\triangle$ teixeira@ufv.br
}

Envío: 2019-08-17

Aceptado: 2020-09-02

First View: 2020-11-20

Publicación: 2020-12-22

RESUMEN: Este trabajo analiza el impacto de eliminar impuestos a los alimentos y bienes de la agricultura para las regiones de Brasil. El impacto de la transferencia de la clase que posee mayor ingreso a la recaudación del gobierno fue también evaluada. Se observaron impactos positivos en el bienestar familiar, pero una caída en el bienestar del gobierno. Considerando la transferencia de ingresos, la cuenta del Estado es restablecida con una disminución del bienestar del sector privado, siendo una política efectiva. 
Palabras clave: política fiscal; inequidad; redistribución de ingresos; bienestar económico

\begin{abstract}
The present paper analyzes the impact of eliminating taxes on food and agricultural consumption goods for the regions of Brazil. The impacts of income transfers from higher-income classes on government revenue were evaluated, as well. The first results show an aggregate positive household welfare impact, despite a fall in government welfare. After income transfers, the government account is reestablished with a subtle private welfare decrease, being an effective income transfer policy.
\end{abstract}

Keywords: fiscal policy; inequality; income redistribution; welfare

\title{
INTRODUCCIÓN*
}

Con el fin de cumplir con sus funciones, ofrecer servicios y financiar las diversas actividades, el gobierno requiere de recursos públicos. Para eso, de acuerdo con Souza (2012), el gobierno utiliza ganancias generadas por el sector privado y las familias que no resultan de las actividades directas que realiza el Estado. Es decir, lo realiza por la tributación, que es donde el Estado posee la función de recaudar tributos.

La Constitución Federal de 1967 consideraba que los estados brasileños debían liberar la primera necesidad de impuesto sobre la Circulación de Mercadería y Servicios, antiguo ICM, sin embargo, la Constitución de 1969 no mantuvo tal disposición legal. La edición reciente de la Constitución, de 1988, no habla sobre la necesidad de liberar el impuesto sobre productos básicos, pero considera que, una vez que se trate de mercadería de la canasta básica, las tarifas deben ser más blandas y, por otro lado, se aplicarán tarifas mayores a productos superfluos (Frasson, 2015).

El hecho es que la configuración del sistema tributario brasileño penaliza a los más pobres por privilegiar el impuesto sobre consumo de bienes y servicios, tales como remedios, alimentos y vestimenta. De acuerdo con Pintos-Payeras (2010), el 10\% de los más pobres pagan, proporcionalmente a su renta, un $44.5 \%$ más impuestos que el $10 \%$ de los más ricos de la población brasileña. La carga tributaria incide en el 53.9 \% de la renta de las familias que reciben hasta dos salarios mínimos, sin embargo, en las familias más ricas, con hasta treinta salarios mínimos, incide sobre el $36.6 \%$ de la renta. Brasil es el tercer país en el ranking de los países con mayores cargas tributarias sobre bienes y servicios, con el $15.40 \%$, un $3.2 \%$ por encima del valor promedio de los países que constituyen la Organización para la Cooperación y el Desarrollo Económico, OCDE (Gomes, 2016).

De acuerdo con la Investigación de Presupuesto Familiar (POF) 2008-2009 (IBGE, 2011), cuanto menor es la línea de renta de las familias mayor es la proporción de la renta gastada en alimentos. Los gastos en los ítems de alimentos representan el 16.1 \% de los gastos totales de las familias brasileñas y el $19.8 \%$ de los gastos de consumo. Cuando se observa

Este trabajo fue realizado con el apoyo de la Coordinación de Perfeccionamiento del Personal de Educación Superior-Brasil (CAPES) -Código de Financiamiento 001- y el Consejo Nacional de Desarrollo Científico y Tecnológico (CNPQ).

Los autores agradecen el apoyo del Instituto de Políticas Públicas y Desarrollo Sostenible (IPPDS-UFV). 
la incidencia en los diferentes niveles de renta, la diferencia se torna más visible, las familias con renta de hasta $\mathrm{R} \$ 830.00$ gastan el $27.8 \%$ de la renta en alimentación, mientras que las familias con rentas superiores a $\mathrm{R} \$ 10375.00$ gastan el 8.5\% de la renta con los mismos gastos. Por eso, la carga tributaria brasileña puede contribuir a elevar la disparidad de la renta observada entre las familias ricas y pobres en el país.

Considerando las funciones económicas del gobierno (asignación de recursos, estabilización económica y redistribución del ingreso), se cuestiona la efectividad de su configuración actual para lograr estos objetivos, dado el carácter progresivo de los impuestos. ¿Existe alguna forma de mitigar los impuestos para las familias menos favorecidas y lograr la función distributiva del gobierno? Una vez que se redujera la tasa impositiva sobre los bienes de consumo de los hogares, en particular los productos alimenticios y agrícolas, se produciría la justicia económica. Los impuestos a la renta, que son de carácter progresivo, actuarían de forma anticíclica para paliar una posible recesión económica.

Por lo anterior, este artículo analiza una reforma tributaria para eliminar del ICMS ${ }^{1}, \mathrm{IPI}^{2}$ e ISS ${ }^{3}$ en el consumo final de productos agrícolas y alimentos, que constituyen una gran parte del consumo de las familias más pobres, y así analizar el impacto en el bienestar económico de las regiones brasileñas. Además, es importante analizar los impactos en el PIB $^{4}$ y en los gastos del gobierno. El motivo de este segundo análisis es que, sumado a que las regiones tienen grandes diferencias socioeconómicas, el $\mathrm{ICMS}^{5}$ es de competencia estadual, así, sus impactos difieren en cada región brasileña.

Un factor importante para considerar es la imposición de grandes fortunas al saldo de las cuentas públicas. Este mecanismo sería menos injusto para la población, ya que sería consistente con la situación económica de cada familia, por lo que la carga tributaria tendría un impacto consistente. Es posible analizar un escenario donde las familias más adineradas transfieren ingresos al gobierno, mientras se eliminan los impuestos a los alimentos y productos agrícolas. La política reduciría la desigualdad generada por la configuración fiscal brasileña y serviría como estabilizador económico.

El presente estudio tiene como objetivo analizar el carácter redistributivo de una política de exención de impuestos para los sectores agrícola y alimentario en la economía brasileña. Para comprender el mecanismo de encadenamiento de dicha política, se utilizó un Modelo de Equilibrio General Computable para Brasil (PAEG), analizando tres escenarios. En un

1. Se cobra en cada estado en la primera transacción de venta de una mercancía, luego de eso, en la circulación de la mercadería, el impuesto se grava únicamente sobre el valor agregado, con base en la transacción anterior.

2. Recogido en la fuente de producción de los bienes, se incorpora al precio de estos, siendo pagado por el consumidor en el momento de la compra. Se aplica proporcionalmente al precio del producto, a tipos variables.

3. El impuesto sobre servicios de cualquier naturaleza, bajo la jurisdicción de los municipios y del Distrito Federal, tiene como hecho imponible la prestación de servicios, aunque estos no constituyan una actividad preponderante del prestador.

4. Producto interno bruto.

5. El impuesto a los servicios (ISS) se fusionó en un solo impuesto, el ICMS -Constitución federal de 1988-. 
primer momento, el impacto en el bienestar de las regiones se analiza considerando los gobiernos regionales y el gobierno federal, sin movilidad de factores entre las regiones, permitiendo analizar los impactos solo dentro de las regiones de forma aislada.

En un segundo paso, se incorpora al análisis la movilidad de factores (capital y trabajo), lo que permite analizar la competencia de estos factores entre regiones. El desglose de las familias en diferentes clases de ingresos en las regiones nos permite analizar el impacto de la política simulada en las familias (de las más pobres a las más ricas) en cada región por separado. El ejercicio final es considerar que las clases de ingresos más altos serán responsables de mantener la actividad del gobierno en la economía, a través de la transferencia de ingresos. Luego se analiza el carácter redistributivo de la política simulada. En este tercer escenario, el objetivo es garantizar el consumo del gobierno, una vez que se espera una pérdida de ingresos de los impuestos que nos apunta al aumento del bienestar de las familias en las primeras clases de ingresos.

\section{REVISIÓN DE LITERATURA}

La política fiscal se refiere al conjunto de medidas mediante las cuales el gobierno recauda ingresos y realiza gastos. El gobierno interviene en la economía con el objetivo de estabilizar las actividades económicas, estimular el crecimiento y mejorar el bienestar. La intervención pública se resume en los tres aspectos principales: eficiencia, equidad y estabilización. Así, el crecimiento económico (estable) y la mejora del bienestar son objetivos públicos, que deben alcanzarse con equidad y una justa distribución del ingreso en la sociedad (Silva, 2015).

Rivero y Jiménez (2008) reiteran que el crecimiento económico inducido por la tributación dependerá básicamente de los efectos de los impuestos en las decisiones de los agentes, así como de los efectos de las políticas de gasto sobre los ingresos tributarios. En principio, una reducción de impuestos aumenta el retorno de la inversión y, por tanto, estimula la acumulación, la innovación y el desarrollo, aumentando así la tasa de crecimiento. Para Myles (2009), un aumento de un determinado impuesto puede tener un impacto significativo en la decisión de los agentes. Sin embargo, los efectos de la tributación no son los mismos en todos los contextos, regiones o países, ya que las consecuencias sobre las decisiones y, a su vez, sobre el crecimiento pueden diferir notablemente.

Por tanto, para lograr un sistema tributario eficiente, tanto desde el punto de vista social como económico, es fundamental recurrir al principio del beneficio, para que la estructura sea más eficiente y justa (Além y Giambiagi, 1999). Por lo tanto, los impuestos deben estar constituidos de tal manera que a cada agente se le cobre según su capacidad para sostener la carga tributaria. Este principio permite que el uso y los beneficios que generan los bienes y servicios otorgados a la sociedad no sean discriminatorios ni exclusivos (Mochón, 2007).

El gobierno también interviene en la economía con el objetivo de brindar información concreta a los consumidores debido a fallas del mercado que llevan a los consumidores a tomar decisiones que no son las ideales. Si bien es importante brindar más información a la sociedad, gravar los alimentos básicos perjudica a los más pobres. Griffith y O’Connell (2010) destacan que gravar la comida reduce el bienestar de quienes la consideran fundamental. Los hogares de bajos ingresos tienden a gastar una mayor proporción de sus ingresos en 
alimentos, por lo que terminan pagando una mayor proporción de sus ingresos en impuestos. Leicester y Windmejer (2010), sin embargo, afirman que, considerando las características nutricionales, un impuesto a las grasas en los alimentos sería regresivo.

Un problema con los impuestos que se dirigen a componentes específicos de los alimentos (grasa o azúcar, por ejemplo) es que los alimentos se componen de varios nutrientes. La segmentación de un mal nutriente con un impuesto puede lograr reducir los niveles de consumo de ese nutriente, pero puede tener la consecuencia no intencionada de incrementar el consumo de otro mal nutriente si están correlacionados negativamente.

Smed, Jensen y Denver (2007) simulan el impacto de una serie de impuestos sobre los nutrientes individuales y descubren que pueden tener consecuencias indeseables para la demanda de otros nutrientes. Esto significa que un impuesto a los alimentos no es deseable, ya que corre el riesgo de poner en peligro los objetivos del gobierno de redistribuir la riqueza. Lo que importa para la redistribución no es si un impuesto en particular es regresivo o no, sino la progresividad del sistema tributario y de beneficios en su conjunto. Si un impuesto es regresivo, el gobierno puede compensar a las familias de bajos ingresos utilizando otras partes del sistema de impuestos y beneficios (Griffith y O'Connell, 2010).

Azevedo et al. (2014) realizan un estudio específico para los estados y municipios brasileños y concluyen que una política fiscal más estricta, medida por cambios en el saldo primario cíclicamente ajustado, no se asocia con un aumento en las medidas de desigualdad en el período 1995 a 2011. En contraste, Chu, Davoodi y Gupta (2000) y Woo et al. (2013) consideran que la desigualdad en la distribución del ingreso se puede explicar por el nivel, la regresividad de los impuestos y las políticas de gasto de los gobiernos. Así, los impuestos directos y el gasto social tienden a mejorar la distribución del ingreso de la economía, mientras que los impuestos indirectos tienden a incrementar la desigualdad.

Longo y Troster (1993) destacan que una redistribución del ingreso ayuda a reducir las ineficiencias del mercado. Las transferencias, los impuestos y los subsidios son formas que le permiten al gobierno llevar a cabo su función distributiva. En términos más generales, es a través de las transferencias que el gobierno fomenta la redistribución de la renta y debe gravar a los agentes de mayores ingresos con mayores cargas, reteniendo recursos para subsidiar a otros con menores ingresos.

Ball et al. (2013) consideran que, entre 1978 y 2009, la consolidación fiscal en 17 países de la OCDE tuvo efectos significativos en la distribución del ingreso. Los autores destacan el aumento de la desigualdad, la disminución del trabajo (salario) en la formulación de ingresos y el aumento del desempleo en el largo plazo. La evidencia también sugiere que los ajustes basados en el gasto tuvieron, en promedio, mayores efectos distributivos que los ajustes basados en impuestos.

Utilizando modelos estáticos y dinámicos para datos de panel, Fernandes, Cunha y Vasconcelos (2018) analizan el impacto de la política fiscal sobre la desigualdad en la distribución del ingreso en Brasil. Los autores buscaron identificar qué categorías de gasto público contribuyeron a la reducción de la desigualdad, de 2004 a 2014. Los resultados indican que un mayor gasto en asistencia social y seguridad social promueve niveles más bajos de desigualdad.

Hollar y Cubero (2010) afirman que los impactos de la política fiscal sobre la distribución del ingreso en países desarrollados y en desarrollo tienden a ser similares. Goñi, López 
y Servén (2008), sin embargo, señalan que la recaudación de impuestos en los países latinoamericanos es menor que en los países europeos. Los autores también señalan que, a pesar de la menor colaboración, tanto personal como corporativa, el impuesto sobre la renta de las clases altas se redujo del 49.5\% en 1985 al 30\% en 2004.

Para el caso de Brasil, el estudio de Varsano et al. (1998) aborda básicamente las mismas características señaladas por Goñi, López y Servén (2008), pero el primero afirma que, dada la crisis fiscal del Estado brasileño, en el momento del estudio, se descarta cualquier política que redujera la recepción del Estado. Dado el impuesto al consumo como la cantidad más segura que recibe el Estado central y acompañado de incertidumbres a través de un mayor impuesto a la renta, este cambio quedó en un segundo plano.

Un aumento de la tributación de las personas de mayores ingresos es preocupante en cuanto a su cambio de comportamiento ante la reducción de sus ingresos efectivos. Un posible comportamiento adverso es la no transición del agente económico a una clase de ingresos superior, ya sea variando la fuente de ingresos que se declara, cambiando el número de hijos (hay una deducción del impuesto sobre la renta por cada hijo) y otros. Sáez (2001) indica que una tributación más eficiente debería incrementar los ingresos, pero con cierto límite.

Por el contrario, Silveira, Fernandes y Passos (2019) afirman que todavía hay espacio para una mayor tributación de las clases de mayores ingresos. Cuanto mayor sea el nivel de ingresos de los agentes, mayor será la participación de los ingresos exentos de impuestos. Por tanto, la progresividad del impuesto no se vería afectada. En relación a utilidades y dividendos, la obra mencionada estima una pérdida de hasta $\mathrm{R} \$ 40$, si fueran gravados progresivamente.

Los ingresos entre los estados brasileños también son diversos. Hay ingresos más altos en el sur y sureste y más bajos en el norte y noreste, aunque todos los estados están sujetos a las mismas leyes federales (Piancastelli, Perobelli y Mello, 1996). Al aumentar el impuesto directo, es posible que también haya una redistribución de la renta entre los estados. Como señalan Medeiros, Souza y Castro (2015), a pesar del aumento de los ingresos, su apropiación se debe principalmente a la parte más rica de la población.

\section{METODOLOGÍA}

Para lograr la propuesta se utilizó un Modelo Computable de Equilibrio General, el Proyecto de Análisis de Equilibrio General de la Economía Brasileña (PAEG), un conjunto analítico de equilibrio general estático, multirregional y multisectorial, basado en GTAPinGAMS.

El modelo utiliza la sintaxis del algoritmo Modeling Programming System for General Equilibrium, desarrollado por Rutherford (1999), que representa un modelo de equilibrio general a través de bloques de ecuaciones de producción, demanda y restricciones específicas. El MPSGE transforma esta información en ecuaciones algebraicas, que se procesan en el software GAMS ${ }^{6}$. Las ecuaciones generadas caracterizan condiciones de beneficio cero para

6. General Algebraic Modeling System es un sistema de modelado para programación y optimización matemática que permite modelar muy cerca del lenguaje matemático. 
la producción, equilibrio entre oferta y demanda en los mercados y definición de ingresos para los consumidores del modelo.

En PAEG, la base de datos de la economía brasileña está desagregada en cinco regiones, manteniendo los datos del GTAP $^{7}$ para las otras regiones y datos sobre los flujos comerciales entre Brasil y otras regiones del mundo intactos. Se decidió utilizar dos versiones del modelo, una con posibilidad de dividir al gobierno en dos (Nazareth, 2017) y la versión que permite dividir a las familias en diez clases de ingresos (Wolf, 2017). La Tabla I presenta la agregación del PAEG, con destaque para las regiones y sectores analizados en este estudio.

\section{TABLA I. AGREGACIÓN DE LOS SECTORES CONSIDERADOS EN EL PAEG}

\begin{tabular}{l|l}
\hline \multicolumn{1}{c|}{ SECTORES } & \multicolumn{1}{c}{ REGIONES } \\
\hline Arroz (pdr) & Brasil - Norte \\
\hline Maíz y otros cereales (gro) & Brasil - Nordeste \\
\hline Soja y otras oleaginosas (osd) & Brasil - Centro-Oeste \\
\hline Industria del azúcar (c_b) & Brasil - Sudeste \\
\hline Carne y animales vivos (oap) & Brasil - Sur \\
\hline Leche y lácteos (rmk) & Resto del Mercosur \\
\hline Otros productos agrícolas (agr) & Estados Unidos \\
\hline Alimentos (foo) & Resto del NAFTA \\
\hline Industria textil & Resto de las Américas \\
\hline Vestuario y calzados & China \\
\hline Madera y mobiliarios & Resto del mundo \\
\hline Papelería & \\
\hline Químicos, gomas y plásticos & \\
\hline Manufacturas & \\
\hline Eletricidad, gas y distribución & \\
\hline Comercio & \\
\hline Construcción & \\
\hline Transporte & \\
\hline Administración pública & \\
\hline
\end{tabular}

Fuente: Teixeira, Pereira y Gurgel (2013).

7. Center of Global Trade Analysis (Hertel, 1997). El modelo GTAP tiene una base de datos global y describe patrones bilaterales de comercio, producción, consumo y uso intermedio de bienes y servicios. 
Cada región está representada por una estructura de demanda final, compuesta por el gasto público y privado en bienes y servicios. Los sectores productivos combinan insumos intermedios y factores primarios de producción -capital, trabajo, tierra y recursos naturales- con el fin de minimizar costos, dada la tecnología. La base de datos incluye los flujos comerciales bilaterales entre países y regiones, así como costos de transporte, aranceles de importación e impuestos (o subsidios) de exportación.

En el trabajo de Teixeira, Pereira y Gurgel (2013), se presentan en detalle las variables que representan los niveles de actividades en equilibrio y las variables de precios relativos de bienes y factores. Todas las variables tienen un valor determinado por el modelo, donde cada precio en equilibrio está asociado con la condición de equilibrio del mercado. Los procesos de optimización se describen mediante diferentes relaciones matemáticas que ocurren en el modelo de equilibrio general. Además de estas relaciones, el modelo considera las condiciones de equilibrio entre oferta y demanda en los mercados, beneficio cero y equilibrio entre ingresos y gastos de los agentes, para completar el proceso de balance computacional.

El consumo del sector público combina materias primas nacionales $\left(\operatorname{vdgm}_{\mathrm{ir}}\right)$ e importadas $\left(\right.$ vigm $\left._{\mathrm{ir}}\right)$, a través de una función de elasticidad de sustitución constante (CES), e impuestos $\left(\mathrm{RG}_{\mathrm{ir}}\right)$, para formar la demanda agregada del gobierno $\left(\mathrm{G}_{\mathrm{r}}\right)$, a partir de como sigue:

$$
G_{r}: \sum_{i}\left(v d g m_{i r}+v i g m_{i r}\right)+R_{i r}^{G}
$$

Los ingresos del gobierno tienen en cuenta los ingresos por impuestos indirectos sobre la producción y las exportaciones $\left(\mathrm{RY}_{\mathrm{ir}}\right)$, las importaciones $\left(\mathrm{RM}_{\mathrm{ir}}\right)$, el consumo $\left(\mathrm{RC}_{\mathrm{r}}\right)$, la demanda del gobierno $\left(\mathrm{RG}_{\mathrm{r}}\right)$ y las transferencias de factores de producción $\left(\mathrm{RHH}_{\mathrm{r}}\right)$ del exterior $\left(v b_{r}\right.$.) y transferencias netas entre el gobierno y las familias $\left(v_{t a x_{r}}\right)$. Por tanto, la limitación del gobierno es:

$$
v g m_{r}=\sum_{i} R_{i r}^{Y}+\sum_{i} R_{i r}^{M}+R_{r}^{C}+R_{r}^{G}+R_{r}^{H H}+v b_{r}-v \operatorname{tax}{ }_{r}
$$

El ingreso total del gobierno, dado por transferencias internacionales, transferencias familiares e ingresos tributarios, debe ser igual al gasto público, es decir, vgmr $=G_{r^{r}}$ En el nivel más alto de la canasta de consumo del gobierno, los bienes se combinan en una función de Leontief (la elasticidad de sustitución es igual a cero).

El sector privado combina materias primas nacionales $\left(\mathrm{vdpm}_{\mathrm{ir}}\right)$ e importadas $\left(\mathrm{vipm}_{\mathrm{ir}}\right)$, considerando una elasticidad de sustitución constante (CES), e impuestos $\left(\mathrm{RC}_{\mathrm{ir}}\right)$, para formar el consumo agregado de los hogares, de la siguiente manera:

$$
C_{r}: \sum_{i}\left(v d p m_{i r}+v i p m_{i r}\right)+R_{i r}^{C}
$$


La restricción presupuestaria del agente representativo es igual a la renta de los factores de producción $\left(\right.$ evom $\left._{\mathrm{fr}}\right)$ menos los impuestos sobre la renta de los factores $\left(\mathrm{RHH}_{\mathrm{ir}}\right)$, las transferencias entre el gobierno y las familias $\left(\operatorname{vtax}_{\mathrm{r}}\right)$, con los gastos de consumo $\left(\mathrm{vpm}_{\mathrm{r}}\right)$ y la inversión privada $\left(\operatorname{vim}_{\mathrm{r}}\right)$, de la siguiente manera:

$$
\sum_{f} e v o m_{f r}-R_{r}^{H H}+\operatorname{vtax}_{r}=v p m_{r}+v i m_{r}
$$

El ingreso total del agente representativo, dado por el ingreso de los factores de producción, menos los impuestos sobre los factores, más las transferencias netas entre el gobierno y las familias, debe ser igual al gasto privado $\left(\mathrm{vpm}_{\mathrm{r}}=\mathrm{C}_{\mathrm{r}}\right)$. En la canasta de consumo del agente representativo, los bienes se combinan en una función Cobb-Douglas (la elasticidad de sustitución es igual a uno). A priori, el modelo considera $\operatorname{vtax}_{\mathrm{r}} \mathrm{y} \operatorname{vim}_{\mathrm{r}} \operatorname{como}_{\mathrm{n}}$ exógenos.

El consumo del agente privado puede representarse mediante un problema de minimizar el costo de un nivel dado de consumo agregado, como sigue ${ }^{8}$ :

$$
\min _{\text {ddpm,dipm }} \sum_{s} p y_{i s}\left(1+r t_{i r}^{p d}\right) d d p m_{i r}+p m_{i r}\left(1+r t_{i r}^{p i}\right) d i p m_{i r}
$$

Sujeto a $H_{r}($ ddpm, dipm $)=C_{i r}$

Donde: $\mathrm{py}_{\mathrm{is}}$ es el precio de oferta interno, bruto de impuestos/subsidios indirectos a la producción; $\mathrm{pm}_{\mathrm{ir}}$ es el precio de importación, bruto de impuestos indirectos sobre las importaciones; $\mathrm{rtpd}_{\mathrm{ir}}$ son impuestos sobre el consumo privado dentro de la propia región; y rtpi $\mathrm{i}_{\mathrm{ir}}$ son impuestos sobre el consumo privado originados fuera de la región. Las materias primas

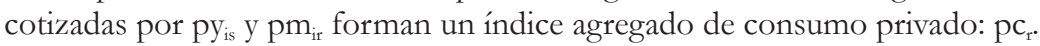

El modelo almacena, en su base de datos, los impuestos específicos de las regiones brasileñas. Por tanto, para representar una reducción de estos impuestos sobre el consumo de los hogares en las regiones brasileñas, basta con eliminarlos de los impuestos al consumo privado dentro y fuera de la región (ver Anexo).

El cierre del modelo considera que la oferta total de cada factor de producción no cambia, pero estos factores son móviles entre sectores dentro de una región. No hay desempleo en el modelo, por lo que los precios de los factores son flexibles. Por el lado de la demanda, las inversiones y los flujos de capital se mantienen fijos, así como el saldo en la balanza de pagos; por tanto, debe haber alteraciones en el tipo de cambio real para acomodar las variaciones entre exportaciones e importaciones en los escenarios analizados. El consumo agregado del agente representativo puede cambiar con cambios en los precios de los bienes, así como los ingresos por impuestos estarán sujetos a cambios en el nivel de actividad y consumo.

8. En estas ecuaciones, las variables de decisión corresponden a los datos iniciales (o «punto de referencia»), con la letra inicial «d» en lugar de la letra «v»». 
El análisis se realizará en tres escenarios:

A) El primer escenario no considera el desglose de familias, con el fin de poder evaluar la movilidad de factores primarios (capital y trabajo) entre regiones, de esta manera se podrá verificar la fuga de factores entre regiones.

Se analiza el impacto sobre el bienestar en las regiones brasileñas y en los principales agregados del PIB, incluyendo el consumo de los gobiernos federal y regional.

B) El segundo escenario considera las clases de ingreso desagregadas en las regiones brasileñas, lo que permite analizar el impacto de la política de eliminación de impuestos sobre el bienestar de las familias en cada región, así como el impacto sobre el PIB.

C) El tercer escenario será considerar el mecanismo de transferencia de las familias más ricas del modelo (clases 8.9 y 10) para analizar los efectos sobre el bienestar de las familias y el PIB.

Cuando se decide permitir la movilidad de factores entre las regiones del modelo, en el primer escenario, no es posible considerar el desglose por clase de ingreso, ya que la activación de estas dos propiedades al mismo tiempo genera mayor complicación en la formulación matemática del modelo y requiere más datos para su correcta representación. Además, al considerar el desglose en diez clases de ingresos, es posible analizar solo el comportamiento de los factores capital y trabajo.

En la versión federal PAEG, los impuestos en la economía se dividen entre el gobierno federal y el gobierno regional, considerando la transferencia neta entre ellos ( $\left.\mathrm{TF}^{\mathrm{RF}}\right)$, la restricción del gobierno en $\operatorname{vgm}_{\mathrm{rF}} \mathrm{y} \mathrm{vmg}_{\mathrm{rR}}$ se distingue de la siguiente manera?

$$
\begin{gathered}
v g m_{r F}=\sum_{i} R_{i r F}^{Y}+R_{r F}^{C}+\sum_{i} R_{i r F}^{M}+R_{r F}^{G F}+R_{r F}^{G R}+R_{r F}^{H H}+T R^{R F}+v b_{r F}-v \operatorname{tax}_{r F} \\
v g m_{r R}=\sum_{i} R_{i r R}^{Y}+R_{r R}^{C}+\sum_{i} R_{i r R}^{M}+R_{r R}^{G F}+R_{r R}^{G R}+R_{r R}^{H H}+T R^{R F}-v \operatorname{tax}_{r F}
\end{gathered}
$$

Por tanto:

$$
\begin{aligned}
& G F_{r}: \sum_{i}\left(v^{i g m_{i r F}}+\operatorname{vigm}_{i r F}\right)+R_{r R}^{G F}+R_{r F}^{G F} \\
& G R_{r}: \sum_{i}\left(v^{G} m_{i r R}+\operatorname{vigm}_{i r R}\right)+R_{r F}^{G R}+R_{r R}^{G R} \\
& C_{r}: \sum_{i}\left(\text { vdpm }_{i r}+\operatorname{vipm}_{i r}\right)+R_{r F}^{C}+R_{r R}^{C}
\end{aligned}
$$

9. Ver Nazareth (2017). El gobierno federal recibe transferencias internacionales, $\mathrm{vb}_{\mathrm{rF}}$. 
En el segundo escenario, el gobierno vuelve a ser uno, y la activación de las diferentes clases de ingresos divide el consumo del agente privado $\left(\mathrm{vdpm}_{\mathrm{ir}} \mathrm{y}\right.$ vipm $\mathrm{vir}_{\mathrm{ir}}$ ) en diez clases en las regiones brasileñas $\left(v_{d p m} f_{\text {ir }} y\right.$ vdpm_ $\left.f_{\text {ir }}\right)$, así como los ingresos por factores primarios. $\left(\right.$ evom_ $\left.f_{\text {fr }}\right)$ e impuestos directos $\left(v \operatorname{vtax} \_f_{r}\right)$, donde $f=1, \ldots, 10$. Además, los productos básicos nacionales (py) y los importados (pm) se combinan para formar un índice de consumo agregado $(\mathrm{pc})$ para cada familia en las regiones brasileñas $\left(\mathrm{pcbra}_{\mathrm{fr}}\right)$. Esto permite que el modelo cambie su formación como agente único para representar diez clases de familias en cada región, separadas por nivel de ingresos, y representar las preferencias de consumo específicas de las diferentes clases.

El desglose por clases de ingresos permite un análisis más preciso de los impactos de las políticas gubernamentales en diferentes estratos sociales, en las cinco regiones brasileñas. Las clases de ingresos consideradas en el modelo son:

Clase 1 - hasta $\mathrm{R} \$ 400.00$;

Clase 2 - de $\mathrm{R} \$ 400,00$ hasta $\mathrm{R} \$ 600.00$;

Clase 3 - de $\mathrm{R} \$ 600,00$ hasta $\mathrm{R} \$ 1000.00$;

Clase 4 - de $\mathrm{R} \$ 1000,00$ hasta $\mathrm{R} \$ 1200.00$;

Clase 5 - de $\mathrm{R} \$ 1200,00$ hasta $\mathrm{R} \$ 1600.00$;

Clase 6 - de $\mathrm{R} \$ 2000,00$ hasta $\mathrm{R} \$ 3000.00$;

Clase 7 - de $\mathrm{R} \$ 3000,00$ hasta $\mathrm{R} \$ 4000.00$;

Clase 8 - de $\mathrm{R} \$ 4000,00$ hasta $\mathrm{R} \$ 5000.00$;

Clase 9 - de $\mathrm{R} \$ 5000,00$ hasta $\mathrm{R} \$ 6000.00$;

Clase 10 - más de R\$6000.00.

En el tercer escenario, se desactiva la opción de distinguir entre gobierno federal y regional, y se agrega una variable al modelo que permite transferencias de ingresos endógenos netos entre las familias más ricas del modelo $(8,9$ y 10$)$ y el gobierno, para mantener constante la actividad del sector público después de un shock, por ejemplo, reduciendo los impuestos indirectos sobre el consumo por parte del consumo privado, esta variable se define como taur. Por tanto, cuando $\mathrm{f}=8,9$ y 10 , tenemos:

$$
\begin{gathered}
v g m_{r}=\sum_{i} R_{i r}^{Y}+\sum_{i} R_{i r}^{M}+R_{r}^{C}+R_{r}^{G}+R_{r}^{H H}+v b_{r}-v \operatorname{tax}_{r}+\operatorname{tau}_{r} \\
\sum_{f} \text { evom }_{f r}-R_{r}^{H H}+v \operatorname{tax}_{r}-\operatorname{tau}_{r}=v p m_{r}+v i m_{r}
\end{gathered}
$$

La variable taur debe asumir un valor en la solución del modelo que equilibre los gastos e ingresos del gobierno luego de cualquier escenario aplicado al modelo, es decir, el ingreso de las familias más ricas se reduce con la misma intensidad que el gobierno necesita para mantener el nivel de consumo del sector público.

El parámetro que calcula el cambio en el bienestar económico es el cambio equivalente (VE) en términos porcentuales. Varian (1992) indica que VE expresa el cambio necesario en el ingreso para mantener el nivel de utilidad, a los precios del equilibrio inicial, cuando el consumidor enfrenta un nuevo conjunto de precios: 
R. WOLF, A. ABOUBACARI MOHAMED, F. SEPÚLVEDA GOMES, A. COSTA GURGEL Y E. CARDOSO TEIXEIRA IMPACTO DE LA ELIMINACIÓN DE IMPUESTOS SOBRE EL CONSUMO DE ALIMENTOS Y PRODUCTOS AGRÍCOLAS EN BRASIL

$$
\mathrm{VE}=\frac{\left(U^{f}-U^{0}\right)}{U^{0}} \mathbf{R A}^{\mathbf{0}}
$$

$\mathrm{U}^{\mathrm{f}}$ representa el nivel de utilidad final, $\mathrm{U}^{0}$ el nivel de utilidad inicial y RA0 los ingresos del agente privado en el saldo inicial.

\section{RESULTADOS Y DISCUSIONES}

$\mathrm{Al}$ principio, buscamos analizar un escenario donde las familias no se dividieran en diferentes clases. Luego de este análisis inicial, se permite la movilidad de factores primarios (capital y trabajo), lo que permite evaluar el impacto de su transferencia entre regiones. Para lograr este resultado, se consideran en el análisis dos tipos de gobierno: uno es el gobierno regional, correspondiente a las regiones brasileñas, y el otro es el gobierno federal. Los impactos de la eliminación de impuestos sobre el consumo de alimentos y productos agrícolas en las regiones brasileñas, sin considerar la movilidad de factores entre regiones, sobre el bienestar de las regiones se muestran en la Tabla II.

TABLA II. IMPACTO DE LA RETIRADA DE LOS IMPUESTOS SOBRE EL CONSUMO DE ALIMENTOS Y PRODUCTOS AGRÍCOLAS EN EL BIENESTAR DE LAS REGIONES BRASILEÑAS (SIN MOVILIDAD DE FACTORES ENTRE LAS REGIONES)

\begin{tabular}{l|c|c|c|c}
\hline \multicolumn{1}{c|}{ REGIÓN } & BIENESTAR & PIB & GOBIERNO_R & GOBIERNO_F \\
\hline Norte & $0.20 \%$ & $0.14 \%$ & $-0.64 \%$ & $-0.21 \%$ \\
\hline Nordeste & $0.17 \%$ & $0.11 \%$ & $-0.55 \%$ & $-0.21 \%$ \\
\hline Centro-Oeste & $0.19 \%$ & $0.17 \%$ & $-0.74 \%$ & $-0.21 \%$ \\
\hline Sudeste & $0.06 \%$ & $0.09 \%$ & $-0.36 \%$ & $-0.21 \%$ \\
\hline Sur & $0.27 \%$ & $0.22 \%$ & $-0.83 \%$ & $-0.21 \%$ \\
\hline
\end{tabular}

Donde:

Gobierno_R es el bienestar del gobierno regional.

Gobierno_F es el bienestar del gobierno federal.

Fuente: Resultados de la investigación.

Dado que el escenario reduce los impuestos sobre el consumo final de todos los hogares, el impacto inmediato es una reducción de los ingresos públicos (federales y regionales), por lo tanto, una reducción de su capacidad de consumo, lo que representa una caída en su bienestar económico. Los mayores impactos se encuentran en las regiones donde hay mayor actividad agrícola y alimentaria. Las regiones Sur (-0.831\%) y Centro-Oeste $(-0.735 \%)$ son aquellas donde el gobierno regional tiene una mayor pérdida de bienestar. 
Por otro lado, la eliminación de los impuestos al consumo significa un aumento del bienestar de los hogares. Las familias más beneficiadas por la política serían las del Sur y Norte, con ganancias en bienestar del $0.273 \%$ y $0.204 \%$, respectivamente. Es evidente que el calentamiento en términos de consumo interno en los sectores agrícola y alimentario aumenta el bienestar en todas las regiones, elevando el PIB, especialmente en las regiones donde estos sectores asumen una mayor participación en las actividades económicas, incluso con la participación de ambos gobiernos en la economía.

La Tabla III muestra el volumen de capital y trabajo transferido entre las regiones, debido a que los factores pueden moverse. Los valores positivos indican que la región recibe un cierto tipo de factor, mientras que la región pierde recursos si el valor es negativo. Por lo tanto, cuanto mayor sea el valor informado en la Tabla III, mayor será el valor recibido para el factor en cuestión. Se espera que las regiones con ventaja comparativa en los sectores favorecidos por la política en relación con las demás reciban un mayor volumen de recursos (capital/trabajo).

\section{TABLA III. IMPACTO DE LA RETIRADA DE LOS IMPUESTOS SOBRE ALIMENTOS Y AGRICULTURA EN LA MOVILIDAD DE FACTORES ENTRE LAS REGIONES BRASILEÑAS}

\begin{tabular}{l|c|c|c|c|c}
\hline \multicolumn{1}{c|}{ FACTOR } & NORTE & NORDESTE & $\begin{array}{c}\text { CENTRO- } \\
\text { OESTE }\end{array}$ & SUDESTE & SUR \\
\hline Trabajo & 0.12 & -0.13 & 1.25 & -0.54 & 0.92 \\
\hline Capital & 0.1 & -0.11 & 1.22 & -0.62 & 0.93 \\
\hline * Variación porcentual
\end{tabular}

Fuente: Resultados de la investigación.

Los resultados indican que la exención de impuestos al consumo final de alimentos y productos agrícolas intensifica la ventaja comparativa de la región del Centro-Oeste como principal productor de alimentos del país, lo que acaba atrayendo capital y mano de obra de otras regiones. Las regiones Sur y Norte también reciben recursos de otras regiones. En este escenario, Sureste y Nordeste pierden factores para las demás regiones, ya que los sectores agroalimentarios son menos expresivos en estas regiones en comparación con las demás.

Los impactos de la eliminación de impuestos sobre el consumo de alimentos y productos agrícolas, considerando la movilidad de factores entre regiones, sobre el bienestar de las regiones se muestran en la Tabla IV.

El gobierno regional del Centro-Oeste se beneficia en términos de bienestar, que se explica fácilmente por el fuerte aumento del PIB, de 1.880 US\$mi y del consumo en esta región. Es decir, con perfecta movilidad, la exención de impuestos al consumo final de alimentos intensifica la ventaja comparativa de la región, que termina atrayendo capital y mano de obra de otras regiones y creciendo hasta revertir la pérdida de recaudación tributaria regional. La región Sur tiene un crecimiento del PIB aún más acentuado que la región Centro-Oeste, que representa 3.9 US\$bn, pero no lo suficiente para revertir la pérdida en la recaudación. 
R. WOLF, A. ABOUBACARI MOHAMED, F. SEPÚLVEDA GOMES, A. COSTA GURGEL Y E. CARDOSO TEIXEIRA IMPACTO DE LA ELIMINACIÓN DE IMPUESTOS SOBRE EL CONSUMO DE ALIMENTOS Y PRODUCTOS AGRÍCOLAS EN BRASIL

TABLA IV. IMPACTO DE LA RETIRADA DE IMPUESTOS SOBRE ALIMENTOS Y AGRICULTURA EN LAS REGIONES BRASILEÑAS (CON MOVILIDAD DE FACTORES ENTRE LAS REGIONES)

\begin{tabular}{l|c|c|c|c}
\hline \multicolumn{1}{c|}{ REGIÓN } & BIENESTAR & PIB & GOBIERNO_R & GOBIERNO_F \\
\hline Norte & $0.331 \%$ & $0.206 \%$ & $-0.570 \%$ & $-0.178 \%$ \\
\hline Nordeste & $0.064 \%$ & $0.005 \%$ & $-0.607 \%$ & $-0.178 \%$ \\
\hline Centro-Oeste & $1.334 \%$ & $1.365 \%$ & $0.133 \%$ & $-0.178 \%$ \\
\hline Sudeste & $-0.443 \%$ & $-0.458 \%$ & $-0.750 \%$ & $-0.178 \%$ \\
\hline Sur & $1.232 \%$ & $1.146 \%$ & $-0.214 \%$ & $-0.178 \%$ \\
\hline
\end{tabular}

Donde:

Gobierno_R es el bienestar del gobierno regional.

Gobierno_F es el bienestar del gobierno federal.

Fuente: Resultados de la investigación.

Esto puede deberse a que los impuestos sobre alimentos y productos agrícolas tienen un mayor peso en la recaudación tributaria del Sur en relación al Centro-Oeste, por lo que la eliminación de la recaudación tributaria por alimentos en el Sur no es reemplazada por la recaudación tributaria de otros sectores a pesar de la mejora de la economía, ya que el Sur es una región más relevante para el consumo en estos sectores, es decir, el Sur está más poblado y gasta más recursos en el consumo de alimentos y productos agrícolas, mientras que el Centro-Oeste es un importante exportador de alimentos al resto del país.

El calentamiento de la economía del Sur significa un mayor aumento del consumo de alimentos que en otras regiones. La región Sudeste presenta una caída en el bienestar económico, debido a que los sectores beneficiados por la reducción de impuestos son menores con relación al resto de sectores de la economía. El impacto negativo se siente en el PIB y en el bienestar del gobierno regional, la región pierde recursos frente a otras donde los sectores agroalimentario y agropecuario son más productivos.

Los impactos de la retirada de impuestos sobre el consumo de alimentos y productos agrícolas en las regiones brasileñas sobre el bienestar de cada clase de ingresos en las regiones se presentan en la Tabla V. Dado que los impuestos sobre los sectores analizados tienden a reducir su precio en relación con el resto de sectores, los resultados sugieren mayores ganancias en bienestar económico cuanto mayor es el consumo de cada clase de los sectores con impuesto reducido, con ganancias en bienestar para las familias y una reducción en el consumo de bienes distintos a los del corte debido a que el precio relativo de los productos alimenticios y agrícolas está bajando.

Se advierte que, en general, las familias de las primeras clases de ingresos son las que presentan una mayor ganancia de bienestar, principalmente en las regiones donde los sectores agrícola y alimentario son más expresivos en relación con los demás sectores de la economía. Las familias más pobres tienen una mayor participación en el consumo de alimentos y productos agrícolas. El resultado muestra que los impuestos sobre el consumo de bienes básicos castigan más a las familias pobres que a las familias ricas. Las familias más pobres gastan una 
R. WOLF, A. ABOUBACARI MOHAMED, F. SEPÚLVEDA GOMES, A. COSTA GURGEL Y E. CARDOSO TEIXEIRA IMPACTO DE LA ELIMINACIÓN DE IMPUESTOS SOBRE EL CONSUMO DE ALIMENTOS Y PRODUCTOS AGRÍCOLAS EN BRASIL

TABLA V. IMPACTO DE LA RETIRADA DE IMPUESTOS SOBRE EL CONSUMO DE ALIMENTOS Y PRODUCTOS AGRÍCOLAS EN EL BIENESTAR ECONÓMICO DE LAS FAMILIAS EN LAS REGIONES BRASILEÑAS

\begin{tabular}{c|c|c|c|c|c}
\hline CLASE DE RENTA & NORTE & NORDESTE & $\begin{array}{c}\text { CENTRO- } \\
\text { OESTE }\end{array}$ & SUDESTE & SUR \\
\hline Clase 1 & $0.574 \%$ & $0.615 \%$ & $0.931 \%$ & $0.251 \%$ & $1.161 \%$ \\
\hline Clase 2 & $0.721 \%$ & $0.506 \%$ & $0.854 \%$ & $0.362 \%$ & $0.963 \%$ \\
\hline Clase 3 & $0.688 \%$ & $0.494 \%$ & $0.847 \%$ & $0.402 \%$ & $0.793 \%$ \\
\hline Clase 4 & $0.637 \%$ & $0.425 \%$ & $0.678 \%$ & $0.347 \%$ & $0.623 \%$ \\
\hline Clase 5 & $0.469 \%$ & $0.740 \%$ & $0.601 \%$ & $0.325 \%$ & $0.734 \%$ \\
\hline Clase 6 & $0.385 \%$ & $0.337 \%$ & $0.597 \%$ & $0.281 \%$ & $0.629 \%$ \\
\hline Clase 7 & $0.350 \%$ & $0.267 \%$ & $0.604 \%$ & $0.212 \%$ & $0.450 \%$ \\
\hline Clase 8 & $0.327 \%$ & $0.189 \%$ & $0.598 \%$ & $0.216 \%$ & $0.431 \%$ \\
\hline Clase 9 & $0.262 \%$ & $0.231 \%$ & $0.353 \%$ & $0.169 \%$ & $0.423 \%$ \\
\hline Clase 10 & $0.169 \%$ & $0.148 \%$ & $0.234 \%$ & $0.126 \%$ & $0.253 \%$ \\
\hline
\end{tabular}

Fuente: Resultados de la investigación.

mayor proporción de los ingresos en alimentos y productos agrícolas que los hogares más ricos en todas las regiones, aunque el precio de estos sectores es más bajo en comparación con otros sectores de la economía. Este hecho es corroborado por Griffith y O'Connell (2010), cuando concluyeron que las familias más pobres destinan una mayor parte de sus ingresos a impuestos.

Es importante señalar que menores ganancias en bienestar no necesariamente significan menor consumo comparativamente, ya que la ganancia en términos de porcentaje en bienestar se refiere a una comparación del consumo inicial y posterior al escenario analizado en la propia clase de ingresos. Por ejemplo, las ganancias de bienestar del $0.251 \%$ en la región Sudeste representan una ganancia de 0.016 US\$bn, mientras que en la región del Centro-Oeste, la ganancia del $0.931 \%$ representa 0.015 US\$bn. Una variación del 1,161\% en el bienestar de la primera clase de ingresos en el Sur corresponde a 0.027 US\$bn, mientras que una variación del $0.615 \%$ en el Nordeste corresponde a 0.044 US\$bn.

Los resultados muestran la importancia de analizar las regiones brasileñas de forma aislada. Las regiones más pobladas pueden mostrar un menor aumento en el bienestar en términos de variación porcentual, pero en términos reales las ganancias son altas, como es el caso del Sureste. Lo mismo ocurre con una región donde pocas familias forman parte de los estratos bajos, por lo que los resultados pueden ser más expresivos, como es el caso del Sur. Lo que se encuentra es que, en todas las regiones, las familias más pobres aumentan su bienestar en relación a los más ricos, el peso de los sectores que sufren recortes de impuestos es mayor para los más pobres en todas las regiones. 
Para mantener el nivel de actividad del gobierno al inicio del equilibrio, que pierde bienestar luego de la política adoptada (ya que pierde recaudación y reduce su consumo), el mecanismo de compensación es activado por las familias más ricas del modelo. Es decir, las familias de las clases de ingresos 8, 9, 10 realizarán transferencias al gobierno, con el fin de restablecer su actividad en la economía. El impacto de esta política en las regiones brasileñas se muestra en la Tabla VI.

\section{TABLA VI. COMPENSACIÓN GENERADA POR LAS CLASES DE RENTA ELEVADAS}

\begin{tabular}{l|c|c|c}
\hline \multicolumn{1}{c|}{ REGIÓN } & $\begin{array}{c}\text { CONSUMO DE LAS } \\
\text { FAMILIAS }\end{array}$ & $\begin{array}{c}\text { CONSUMO DEL } \\
\text { GOBIERNO }\end{array}$ & PIB \\
\hline Norte & $0.004 \%$ & $0.342 \%$ & $0.124 \%$ \\
\hline Nordeste & $-0.007 \%$ & $0.313 \%$ & $0.088 \%$ \\
\hline Centro-Oeste & $-0.004 \%$ & $0.452 \%$ & $0.154 \%$ \\
\hline Sudeste & $-0.016 \%$ & $0.214 \%$ & $0.067 \%$ \\
\hline Sur & $0.019 \%$ & $0.433 \%$ & $0.161 \%$ \\
\hline
\end{tabular}

Fuente: Resultados de la investigación.

La Tabla VI muestra que el bienestar privado en las regiones, en conjunto, es positivo en el Norte y Sur y negativo en las demás regiones. Sin embargo, las variaciones son mínimas y el impacto sobre el PIB es positivo para todas las regiones. El impacto es más significativo en regiones con ventajas comparativas en sectores que sufren recortes de impuestos. Es posible observar que la actividad del gobierno se restablece en la economía, con ganancias en términos de bienestar para el gobierno, por lo que se ha logrado el objetivo trazado en el escenario. La suma del bienestar (agregado) no es muy significativa (ni siquiera negativa) porque estas mismas familias son responsables de una parte considerable del consumo (ya que concentran la mayor parte de los ingresos de las regiones). La Tabla VII presenta los impactos para las clases de ingresos de cada región brasileña.

Los resultados presentados en la Tabla VII indican que el bienestar de las familias desagregadas muestra ganancias para todas las clases de ingresos, con la excepción de las clases de ingresos 8 a 10, que son responsables de mantener la actividad del gobierno. Las transferencias de ingresos de las familias más ricas para que el gobierno equilibre sus cuentas representan un aumento del impuesto sobre la renta. Los impactos en las primeras siete clases de ingresos son los mismos que los presentados en el escenario anterior, ya que nada más que la reducción de impuestos al consumo de alimentos y productos agrícolas afecta a estas familias.

El indicador Agg_1 representa el bienestar agregado de las clases de ingresos 1 a 7 en US\$bn, mientras que el indicador Agg_2 representa el bienestar agregado de las familias en 
R. WOLF, A. ABOUBACARI MOHAMED, F. SEPÚLVEDA GOMES, A. COSTA GURGEL Y E. CARDOSO TEIXEIRA IMPACTO DE LA ELIMINACIÓN DE IMPUESTOS SOBRE EL CONSUMO DE ALIMENTOS Y PRODUCTOS AGRÍCOLAS EN BRASIL

TABLA VII. IMPACTO DE LA RETIRADA DE LOS IMPUESTOS SOBRE EL CONSUMO DE ALIMENTOS Y PRODUCTOS AGRÍCOLAS EN EL BIENESTAR

DE LAS FAMILIAS EN LAS REGIONES BRASILEÑAS, MANTENIENDO EL NIVEL DE CONSUMO DEL GOBIERNO

\begin{tabular}{l|c|c|c|c|c}
\hline $\begin{array}{c}\text { CLASE DE } \\
\text { RENTA }\end{array}$ & NORTE & NORDESTE & $\begin{array}{c}\text { CENTRO- } \\
\text { OESTE }\end{array}$ & SUDESTE & SUR \\
\hline Clase 1 & $0.574 \%$ & $0.615 \%$ & $0.931 \%$ & $0.251 \%$ & $1.161 \%$ \\
\hline Clase 2 & $0.721 \%$ & $0.506 \%$ & $0.854 \%$ & $0.362 \%$ & $0.963 \%$ \\
\hline Clase 3 & $0.688 \%$ & $0.494 \%$ & $0.847 \%$ & $0.402 \%$ & $0.793 \%$ \\
\hline Clase 4 & $0.637 \%$ & $0.425 \%$ & $0.678 \%$ & $0.347 \%$ & $0.623 \%$ \\
\hline Clase 5 & $0.469 \%$ & $0.740 \%$ & $0.601 \%$ & $0.325 \%$ & $0.734 \%$ \\
\hline Clase 6 & $0.385 \%$ & $0.337 \%$ & $0.597 \%$ & $0.281 \%$ & $0.629 \%$ \\
\hline Clase 7 & $0.350 \%$ & $0.267 \%$ & $0.604 \%$ & $0.212 \%$ & $0.450 \%$ \\
\hline Clase 8 & $-0.637 \%$ & $-1.222 \%$ & $-0.742 \%$ & $-0.236 \%$ & $-0.671 \%$ \\
\hline Clase 9 & $-0.437 \%$ & $-0.703 \%$ & $-1.075 \%$ & $-0.202 \%$ & $-0.328 \%$ \\
\hline Clase 10 & $-0.583 \%$ & $-0.201 \%$ & $-0.168 \%$ & $-0.154 \%$ & $-0.264 \%$ \\
\hline Agg_1 & $(0.260)$ & $(0.528)$ & $(0.412)$ & $(0.964)$ & $(0.628)$ \\
Agg_2 & $(-0.256)$ & $(-0.546)$ & $(-0.418)$ & $(-1.122)$ & $(-0.577)$ \\
bienestar & $(0.004)$ & $(-0.018)$ & $(-0.006)$ & $(-0.158)$ & $(0.051)$ \\
\hline
\end{tabular}

Fuente: Resultados de la investigación.

los grupos de ingresos 8 a 10. Se observa que el impacto agregado (negativo) que sufren las familias más ricas del modelo, y responsables de las transferencias al gobierno, es prácticamente lo mismo que las ganancias de bienestar de las familias más pobres. Así, el escenario muestra una excelente redistribución del ingreso, con efectos positivos sobre el PIB, pero con el bienestar agregado de las regiones en su conjunto casi sin cambios (las familias pobres no se apoderan por completo del consumo reducido de los ricos).

En general, al comparar el efecto de la eliminación de impuestos con otros trabajos como Ball et al. (2013); Chu, Davoodi y Gupta (2000), Woo et al. (2013), y Smed, Jensen y Denver (2007), se observan conclusiones similares que refuerzan el uso de este mecanismo para mejorar la distribución del ingreso en la economía, pero vemos un resultado opuesto en Azevedo et al. (2014). Los resultados de los cambios en el impuesto sobre la renta en las clases de mayores ingresos contradicen parcialmente los resultados abordados por Varsano et al. (1998), ya que el presente estudio muestra que la compensación de la pérdida de ingresos del gobierno a través de mayores impuestos a las familias de los ricos resulta ser una buena alternativa para la política de redistribución de ingresos.

El presente estudio muestra que la pérdida de ingresos públicos en el primer escenario se convierte en ganancias de bienestar para todas las clases familiares. También se observa que, debido a la movilidad de factores, las regiones con actividades agrícolas más destacadas se 
ven beneficiadas tanto por la reducción del impuesto, que aumenta la ganancia de bienestar de las familias, como por el calentamiento económico debido a que los productos alimenticios son más y luego habrá migraciones de capital y trabajo hacia ellos. En el segundo escenario, si bien algunas regiones muestran una pérdida de bienestar, el ejercicio muestra que las familias en su conjunto se ven beneficiadas por la política de reducción de impuestos, solo el gobierno sufre pérdida de bienestar, con excepción del Centro-Oeste.

Con el aumento del impuesto directo en las clases altas, un posible comportamiento adverso por parte del agente económico sería un intento de evadir impuestos, pero el trabajo no considera esta posibilidad. Font, Spanakos y Bordin (2004) señalan que Brasil tiene algunos impuestos en cascada y su retiro implicaría incertidumbre en relación con el presupuesto del gobierno, lo que justifica, por tanto, una fuente de recaudación a través de impuestos directos. Además, es necesario un escenario político más estable con un superávit primario que permita la reducción del gasto público antes de un cambio en la política fiscal. Dado que el consumo del gobierno se mantiene a través de la transferencia de las familias más ricas $(8,9$ y 10), en el tercer escenario, la transferencia de bienestar de estas a las demás es evidente. El saldo de bienestar agregado a pesar de ello es positivo o con un impacto negativo bajo, lo que revela una política de transferencias eficiente, con impactos positivos en la desigualdad de ingresos.

\section{CONCLUSIONES}

El principal interés del estudio fue investigar los cambios en el bienestar de los hogares mediante la eliminación de los impuestos especiales sobre alimentos y productos agrícolas. Estudios preliminares indican que aumentaría el bienestar de las familias de menores ingresos, ya que gran parte de su salario se gasta en estos rubros. En la primera simulación, sin movilidad de factores entre regiones, hubo un aumento del bienestar en todas las clases de ingresos, siendo las clases con menores ingresos las más beneficiadas. Además, el valor de la variable que representa el PIB aumentó, mientras que el gasto público necesariamente tuvo que disminuir, a medida que disminuyeron los ingresos por impuestos.

En cuanto a la desigualdad de ingresos, se puede decir que ha disminuido, puesto que las clases de ingresos con salarios más bajos tuvieron una mayor ganancia en bienestar que aquellas otras clases cuyos salarios eran más altos.. Sin embargo, al observar la movilidad de capital entre regiones, los resultados de la simulación fueron de un movimiento de capital y trabajo a regiones que ya eran ricas, lo que indica un aumento en la desigualdad de ingresos regional, contrastando así con el resultado de la disminución de la desigualdad de los ingresos en general.

Para restablecer el gasto público, se simuló la transferencia de ingresos de las clases altas junto con la reducción de impuestos. En esta simulación, hubo una reducción aún más marcada en la desigualdad de ingresos entre clases a nivel agregado. A nivel regional, hubo pocos cambios en comparación con el escenario base que se utilizó en la comparación, es decir, el bienestar agregado de las regiones ha cambiado relativamente poco. Este resultado indica la gran importancia de la tributación sobre la renta, reflejando su impacto positivo en la reducción de la desigualdad de ingresos.

Aumentar el consumo de los hogares equivale a una transferencia de efectivo y reducir los ingresos del gobierno solo implica un gasto equivalente para este propósito. Este 
resultado es muy positivo si se considera la rapidez de transferencia y el bajo costo administrativo para el gobierno, utilizando así el recurso recolectado de manera económicamente eficiente.

Si bien el tema de la estabilización no está presente en el análisis, considerar una transferencia de las clases de mayores ingresos al gobierno tiene un doble mérito: objetivos distributivos e ingresos. A pesar de la ganancia en bienestar agregado, casi no hay cambios y una generación de ingresos transfiere ingresos a las familias más pobres.

De esta manera, se concluye que la cuestión del impuesto progresivo de hecho reduce la desigualdad de ingresos y mejora el bienestar agregado. Por lo tanto, eliminar los impuestos a los productos agrícolas y alimentarios y, al mismo tiempo, inducir un mecanismo similar a un impuesto progresivo mejora la distribución del ingreso, hay un impacto muy sutil en el bienestar agregado y una mejora en los ingresos del gobierno.

\section{BIBLIOGRAFÍA}

Alavuotunki, K., Haapanen, M. y Pirttilä, J. (2019). The effects of the value-added tax on revenue and inequality. The Journal of Development Studies, 55(4), 490-508.

Além, A. C. y Giambiagi, F. (1999). Finanças públicas: teoria e prática no Brasil. Rio de Janeiro: Editora Campus.

Andrade, E. (2004). Externalidades. En P. Arvate y C. Biderman (Orgs.), Economia do setor público no Brasil (pp. 16-33). São Paulo: Elsevier.

Azevedo, J. P., David, A. C., Bastos, F. R. y Pineda, E. (2014). Fiscal adjustment and income inequality: subnational evidence from Brazil. The World Bank.

Ball, L. M., Furceri, D., Leigh, M. D. y Loungani, M. P. (2013). The distributional effects of fiscal consolidation (No. 13-151). International Monetary Fund.

Baracho, F. y Riani, F. (2007). Economia do setor público: uma abordagem introdutória. São Paulo: Atlas.

Chu, M. K. Y., Davoodi, M. H. R. y Gupta, M. S. (2000). Income distribution and tax and government social spending policies in developing countries (No. 0-62). International Monetary Fund.

Fernandes, C. B. S., Cunha, M. S. y Vasconcelos, M. R. (2018). Impactos da política fiscal na desigualdade da distribuição de renda: Uma análise para as unidades da federação brasileiras. Encontro da Associação Nacional dos Centros de Pós-Graduação em Economia - Sul. Curitiba-PR.

Font, M. A., Spanakos, A. P. y Bordin, C. (Eds.). (2004). Reforming Brazil. Oxford: Lexington Books.

Frasson, E. R. (2013). ICMS e sua interpretação literal diante da bermenêutica jurídica: uma análise sobre o benefício fiscal da cesta básica, previsto no Artigo 11, anexo 2 do regulamento do ICMS de Santa Catarina. (Trabalho de Conclusão de Curso não publicado). Universidade do Extremo Sul Catarinense - UNESC. Criciúma - SC.

Gomes, M. (2016). As distorções de uma carga tributária regressiva. Desafios do desenvolvimento, São Paulo, 12, 36-43.

Goñi, E., López, J. H. y Servén, L. (2008). Fiscal redistribution and income inequality in Latin America. The World Bank.

Griffith, R. y O’Connell, M. (2010). Public policy towards food consumption. Fiscal Studies, 31(4), 481-507.

Hertel, T. W. (1997). Global trade analysis: modelling and applications. Cambridge University Press.

Hollar, I. V., y Cubero, R. (2010). Equity and fiscal policy: the income distribution effects of taxation and social spending in Central America. IMF Working Papers. 
R. WOLF, A. ABOUBACARI MOHAMED, F. SEPÚLVEDA GOMES, A. COSTA GURGEL Y E. CARDOSO TEIXEIRA IMPACTO DE LA ELIMINACIÓN DE IMPUESTOS SOBRE EL CONSUMO DE ALIMENTOS Y PRODUCTOS AGRÍCOLAS EN BRASIL

Instituto Brasileiro de Geografia e Estatística - IBGE (2011). Pesquisa de Orçamento Familiares 2008-2009. Disponível em: https://biblioteca.ibge.gov.br/visualizacao/livros/liv50063.pdf.

Leicester, A. y Windmeijer, F. (2004). The 'fat tax': economic incentives to reduce obesity (Briefing No. 49). The Institute for Fiscal Studies.

Longo, C. A. y Troster, R. L. (1993). Economia do Setor Público. São Paulo: Atlas. 202 pp.

Medeiros, M., Souza, P. H. G. F. D. y Castro, F. Á. D. (2015). A estabilidade da desigualdade de renda no Brasil, 2006 a 2012: estimativa com dados do imposto de renda e pesquisas domiciliares. Ciência \& Saúde Coletiva, 20, 971-986.

Mochón, F. (2007). Princípios de economia. São Paulo: Pearson Prentice Hall. 328 pp.

Monteiro, J. C. B. (2014). A relação da bistória da bumanidade e os tributos. Portal Tributário, 2014. Disnponível em: http://www.portaltributario.com.br/artigos/relacao-da-historia-humanidade-etributos.htm.

Myles, G. D. (2009). Economic Growth and the Role of Taxation - Theory (No. 713). Paris, France: Organization for Economic Co-operation and Development.

Nazareth, M. S. (2017). Federalismo fiscal market-preserving: uma análise de equilibrio geral computável para o Brasi.l (Tese de Doutorado). Universidade Federal de Viçosa - UFV. Viçosa - MG.

Nelson, M., Erens, B., Bates, B., Church, S. y Boshier, T. (2007). Low income diet and nutrition survey (vol. 3). London: TSO.

Piancastelli, M., Perobelli, F. S. y Mello, G. V. (1996). Imposto de Renda Pessoa Física (IRPF) - Redistribuição da Carga Tributária e Elasticidades (Texto para discussão 451). Brasília: IPEA.

Piketty, T. y Saez, E. (2013). Optimal labor income taxation. In Handbook of public economics (vol. 5, pp. 391-474). Elsevier.

Pintos-Payeras, J. (2010). Análise da progressividade da carga tributária sobre a população brasileira. Pesquisa e Planejamento Econômico, 40(2), 153-186.

Pires, V. (1996). Estado, Mercado e Tributação. Piracicaba: UNIMEP. 124 pp.

Rivero, F. J. D. y Jiménez, F. J. S. (2008). Impuestos y crecimiento económico: Una panorámica. RAE: Revista Asturiana de Economía, (42), 9-30.

Rutherford, T. F. (1999). Applied general equilibrium modelling with MPSGE as a GAMS subsystem: An overview of the modelling framework and syntax. Computational Economics, 14(1-2), 1-46.

Saez, E. (2001). Using elasticities to derive optimal income tax rates. The Review of Economic Studies, 68(1), 205-229.

Schwödiauer, G. y Wenig, A. (1990). The impact of taxation on the distribution of wealth in an economy with changing population. Journal of Population Economics, 3(1), 53-71.

Silva, J. M. A. (2015). Análise Macroeconômica e Avaliação Governamental. Santa Maria: Editora UFSM. $311 \mathrm{pp}$.

Silveira, F. G., Fernandes, R. C. y Passos, L. (2019). Benefícios fiscais do imposto sobre a renda da pessoa física e seus impactos redistributivos. Brasília: IPEA.

Smed, S., Jensen, J. D. y Denver, S. (2007). Socio-economic characteristics and the effect of taxation as a health policy instrument. Food Policy, 32(5-6), 624-639.

Souza, J. M. (2012). Economia Brasileira. São Paulo: Pearson Education, 2102. 149 pp.

Teixeira, E. C., Pereira, M. W. G. y Gurgel, A. C. (2013). A estrutura do PAEG. Campo Grande - MS: Life Editora.

Varsano, R., Pessoa, E. D. P., Silva, N. L. C. D., Afonso, J. R. R., Araujo, E. A. y Ramundo, J. C. M. (1998). Uma análise da carga tributária do Brasil. Brasília: IPEA.

Wolf, R., Pereira, M. W. G., Teixeira, E. C., Gurgel, A. C. y Higano, L. T. M. (2018). Efeitos do programa bolsa família sobre o bem-estar econômico das famílias nas macrorregiões brasileiras: uma análise de equilíbrio geral computável. Análise Econômica, 36(71). 
Wolf, R (2017). Efeitos do Programa Bolsa Família sobre o bem-estar econômico das famílias nas macrorregiões brasileiras: uma análise de equilibrio geral computável. (Dissertação de Mestrado). Universidade Federal de Mato Grosso do Sul - UFMS. Campo Grande - MS.

Woo, J., Bova, M. E., Kinda, M. T. y Zhang, M. Y. S. (2013). Distributional consequences of fiscal consolidation and the role of fiscal policy: What do the data say? (No. 13-195). International Monetary Fund.

\section{ANEXO}

El parámetro del modelo que representa las tasas de consumo de los hogares en la propia región es el rtpdi, r, que representa el impuesto al consumo de los hogares sobre el bien «i» dentro de la región «t». Los parámetros que representan los impuestos (icms, ipi e iss) que se tomarán del consumo final de las familias son icmsi, j, r, que representa la porción del ICMS del activo «ì por el sector «j» que se origina en la región « $\mathrm{r}$ « e ipi_issi, $\mathrm{j}$, r, que representa parte del IPI + ISS del activo «ì por sector «j» que se origina en la región « escenarios simulados en el modelo PAEG fueron:

$$
\begin{aligned}
& r t p d(\langle p d n\rangle, b r a)=r t p d 0(\langle p d n\rangle, b r a)-i p i \_i s s(\langle p d n\rangle,\langle c f \uparrow, b r a)-i c m s(\langle p d n\rangle,\langle c f\rangle, b r a) ; \\
& r t p d\left(\langle g r o », b r a)=r t p d 0\left(\langle g r o », b r a)-i p i \_i s s(\langle g r o », » c f\rangle, b r a)-i c m s(\langle g r o »,,\langle c f\rangle, b r a) \text {; }\right.\right. \\
& \left.r t p d(« o s d\rangle, b r a)=r t p d 0(« o s d\rangle, b r a)-i p i \_i s s(\langle o s d\rangle, » c f\rangle, b r a\right)-i c m s(\langle o s d\rangle,\langle c f\rangle, b r a) \text {; }
\end{aligned}
$$

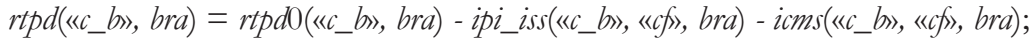

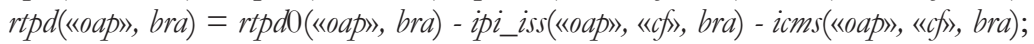

$$
\begin{aligned}
& \left.\left.r t p d(\langle r m k\rangle), b r a)=r t p d 0(\langle r m k\rangle), b r a)-i p i \_i s s(\langle r m k\rangle),\langle c f\rangle, b r a\right)-i c m s(u r m k »\rangle,\langle c f\rangle, b r a\right) \text {; } \\
& \left.r t p d(« a g n\rangle, b r a)=r t p d 0(« a g n\rangle, b r a)-i p i \_i s s(\langle a g n\rangle,\langle c f\rangle, b r a)-i c m s(« a g n\rangle,\langle c f\rangle, b r a\right) \text {; }
\end{aligned}
$$

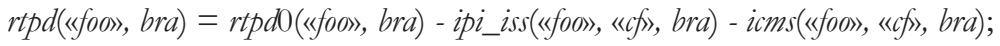

El parámetro del modelo que representa las tasas de consumo de los hogares fuera de la región es el rtpii, r, que representa el impuesto al consumo de los hogares sobre el bien «๖» en la región «t». Luego, para eliminar los impuestos a todos los productos, incluidos los consumidos por familias de la región, originarios de otra región, se agregan las siguientes líneas al código de la simulación:

$$
\begin{aligned}
& r t p i(\langle p d n\rangle, b r a)=r t p i 0\left(\langle p d n », b r a)-i p i \_i s s(\langle p d n »,\langle c f \Uparrow, b r a)-i c m s(\langle p d n »,\langle c f\rangle, b r a) \text {; }\right.
\end{aligned}
$$

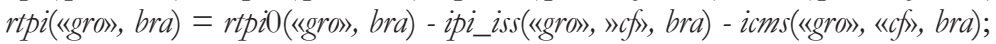

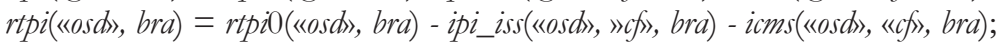

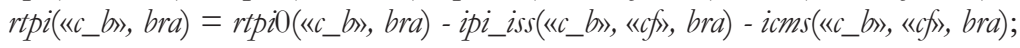

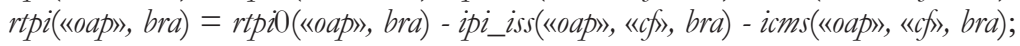

$$
\begin{aligned}
& \left.r t p i(\langle r m k\rangle, b r a)=r t p i 0(\langle r m k\rangle), b r a)-i p i \_i s s(\langle r m k\rangle),\langle c f\rangle, b r a\right)-i c m s(\langle r m k »\rangle,\langle c f\rangle, b r a) \text {; }
\end{aligned}
$$

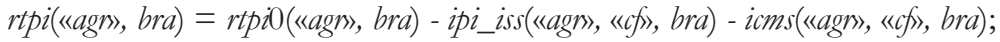

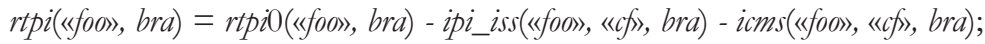

Los escenarios simulados representan que el nuevo impuesto al consumo final de productos importados «cf» en cada región brasileña («bra») será igual a la tasa inicial (rtpdi0) menos impuestos (icms, ipi y iss) en los sectores agroalimentarios. 
